\title{
Limb ischemia and pulmonary artery thrombosis after the ChAdOx1 nCoV-19 (Oxford-AstraZeneca) vaccine: a case of vaccine-induced immune thrombotic thrombocytopenia
}

\author{
Melissa Jones MSc MD, Annie Boisvert MSc MD, Jennifer Landry BSc MD, Paul F. Petrasek MHCM MD
}

Cite as: CMAJ 2021 June 14;193:E906-10. doi: 10.1503/cmaj.210795; early-released May 14, 2021

See related article at www.cmaj.ca/lookup/doi/10.1503/cmaj.210882

A 63-year-old man presented to the emergency department with a 5-day history of a cool, painful left leg, new onset of paresthesia of the left leg and foot, and severe shortness of breath. The patient had no personal or family history of thrombotic events and no personal history or symptoms of arrythmia. He was a nonsmoker with no symptoms or prior documentation of peripheral vascular, coronary or cerebrovascular disease. He had a history of obesity and hypertension controlled with a single agent. Two rapid point-of-care tests were negative for SARS-CoV-2 earlier on the day of presentation (Abbott ID NOW isothermal nucleic acid test). The patient had received his first dose of the ChAdOx $1 \mathrm{nCoV}-19$ vaccine 20 days before the onset of his symptoms.

Upon presentation, we noted that the patient was obese (body mass index 41.8 ) and hypoxic (oxygen saturation $85 \%$ on room air, normal $>90 \%$ ). His left foot was cold, pale and insensate, with decreased motor function and absent arterial Doppler signals (Rutherford IIB acute limb ischemia). ${ }^{1}$ Laboratory investigations showed thrombocytopenia (platelets 36 [normal 150$400] \times 10^{9} / \mathrm{L}$ ), D-Dimer $>10000$ (normal $\left.\leq 50\right) \mathrm{ng} / \mathrm{mL}$ fibrinogenequivalent units, fibrinogen 1.4 (normal 1.6-4.1) $\mathrm{g} / \mathrm{L}$ and international normalized ratio 1.3 (normal 0.9-1.1). A computed tomography angiogram showed left popliteal artery occlusion with no visible tibial vessels, even on delayed imaging. It also showed bilateral segmental pulmonary artery thrombi and thrombus adherent to the wall of the infrarenal aorta (Figure 1).

We started therapeutic anticoagulation with intravenous unfractionated heparin. Emergency surgical thrombectomy of the popliteal artery and all tibial arteries yielded a large amount of thrombus. We infused tissue plasminogen activator into the tibial arteries intraoperatively. We obtained good antegrade and retrograde flow, with marked pedal hyperemia and normal triphasic arterial Doppler signals upon reperfusion. Intraoperatively, the patient was intermittently hypoxic, consistent with his substantial pulmonary artery thrombus.

\section{KEY POINTS}

- Vaccine-induced immune thrombotic thrombocytopenia (VITT) must be considered in any patient presenting with thrombosis and thrombocytopenia after recently receiving a vaccine for SARS-CoV-2 that uses an adenovirus vector (ChAdOx1 nCoV-19 Oxford-AstraZeneca or Ad26.COV2.S Johnson \& Johnson-Janssen).

- The clinical presentation and pathobiology of VITT are similar to those of heparin-induced thrombocytopenia (HIT), but in VITT, an immunoglobulin $\mathrm{G}$ (IgG) antibody against platelet factor 4 triggers platelet activation, thrombosis and thrombocytopenia.

- Commonly used rapid assays for HIT, such as the latexenhanced immunoassay, can be falsely negative in patients with VITT; however, enzyme-linked immunosorbent assays for HIT and serotonin-release assays can be used to detect and confirm VITT, respectively.

- If VITT is suspected, heparin should be discontinued and replaced with an alternate anticoagulant; platelet transfusion should be avoided and intravenous immunoglobulin should be considered in patients who present with clinically worrisome features.

Our patient's postoperative course was complicated by severe hypoxia, thrombocytopenia and substantial surgical site bleeding, for which he received several transfusions of platelets and red bloods. After 24 hours, forefoot hyperemia evolved into cyanosis, with subsequent infarction of the toes, despite therapeutic intravenous heparin (partial thromboplastin time 60-90 [normal $\leq 39$ ] s). We did not observe any clinical sign of compartment syndrome. We performed a rapid assay for heparin-induced thrombocytopenia (HIT) 3 times and all results were negative (HemosIL HIT-Ab ${ }_{(\mathrm{PF}-\mathrm{H})}$ latex-enhanced immunoassay). The patient had no previous heparin exposure. An antiphospholipid antibody screen for anticardiolipin, anti- $\beta 2$ glycoprotein and lupus anticoagulant, and assays for extractable nuclear antigen, antinuclear 

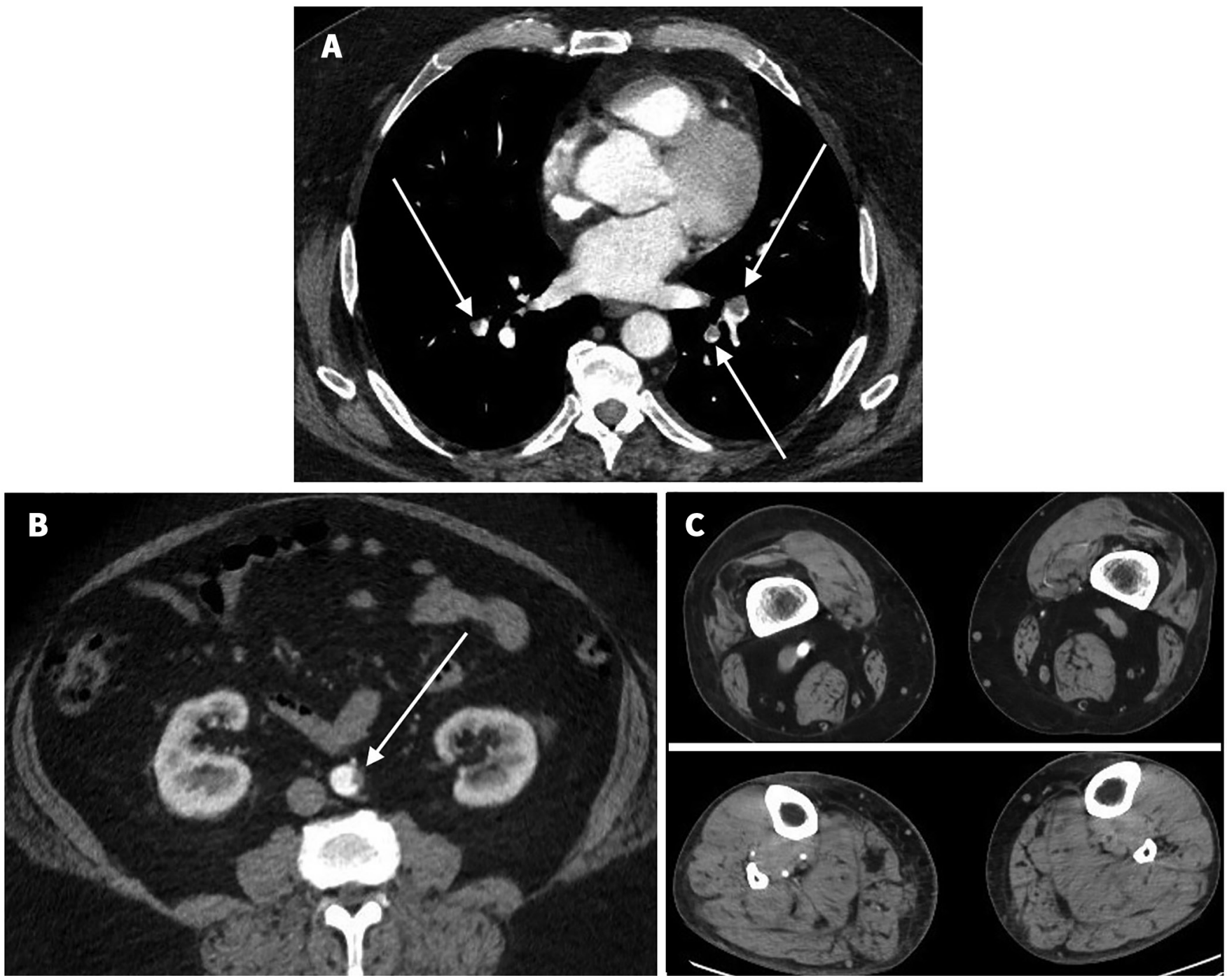

Figure 1: Computed tomography angiograms from a 63-year-old man with vaccine-induced immune thrombotic thrombocytopenia, showing (A) bilateral pulmonary artery thrombus, (B) infrarenal aortic thrombus and (C) normal contrast enhancement of the nonischemic popliteal (top right) and tibial arteries (bottom right) in the right leg, with no enhancement of the corresponding popliteal (top left) and tibial (bottom left) arteries in the ischemic left leg.

antibody, release factor and JAK2 V617F mutation were negative. A postoperative echocardiogram did not show a clinically important patent foramen ovale, which we considered ruled out paradoxical embolism.

We suspected vaccine-induced immune thrombotic thrombocytopenia (VITT) based on ongoing postoperative bleeding, new digital thrombosis and thrombocytopenia that did not respond to platelet transfusion. We replaced unfractionated heparin with fondaparinux (10 mg/d subcutaneously, the dose recommended for body weight $>100 \mathrm{~kg}$ ) and gave intravenous immunoglobulin $(1 \mathrm{~g} / \mathrm{kg} / \mathrm{d})$ for 2 consecutive days (Gammunex). Subsequently, the patient's thrombocytopenia rapidly improved (Figure 2) and the bleeding at his surgical site stopped.

We confirmed VITT on postoperative day 11 by an enzymelinked immunosorbent assay (ELISA) that detected platelet factor 4 (PF4)-immunoglobulin complexes in the patient's serum, and by a serotonin-release assay that showed platelet activa- tion when PF4 was added to the patient's serum, without heparin (McMaster University Platelet Immunology Laboratory, Hamilton, Ont.). On postoperative day 15, arteriograms confirmed partial rethrombosis of the tibial circulation. Aspiration thrombectomy helped to clear this rethrombosis (Figure 3), resulting in substantial reperfusion of the forefoot. We have planned a transmetatarsal amputation once the forefoot fully demarcates (Figure 4).

\section{Discussion}

Vaccine-induced immune thrombotic thrombocytopenia is a syndrome that was first reported in April 2021 among patients who had recently received the ChAdOx1 nCoV-19 vaccine. ${ }^{2-7}$ It closely resembles HIT, in which antibodies, typically immunoglobulin $\mathrm{G}$ (IgG), are produced against circulating complexes of PF4 and endogenous heparin. ${ }^{8-10}$ Platelets neutralize endogenous heparin 

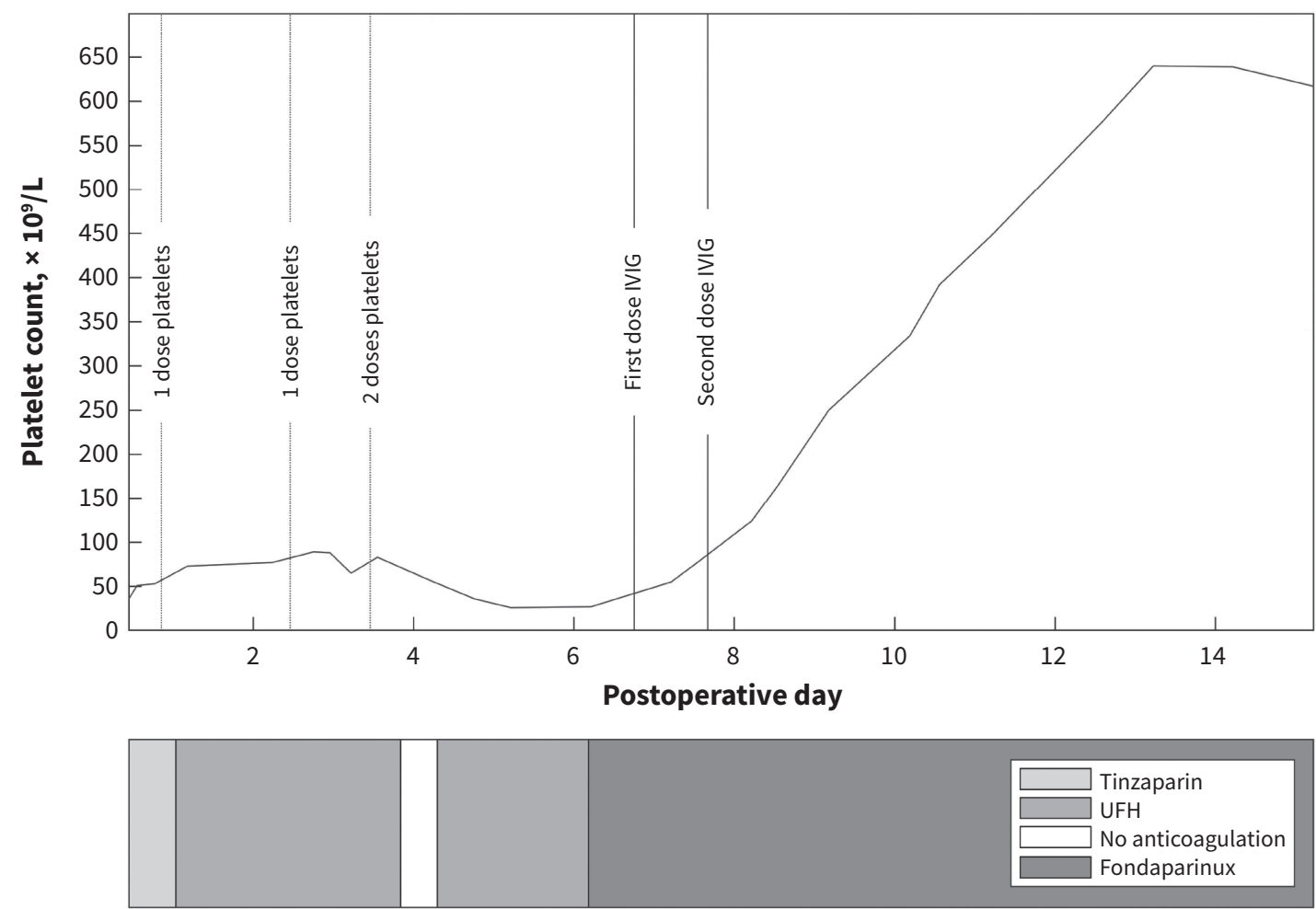

Figure 2: Platelet count and anticoagulation during the patient's clinical course of vaccine-induced immune thrombotic thrombocytopenia. The platelet count was nonresponsive to platelet transfusion. Substantial recovery of platelet count occurred following administration of intravenous immunoglobulin (IVIG). Note: UFH = unfractionated heparin.

and regulate hemostasis by releasing PF4, a 70-amino acid protein that binds heparin. In HIT, the IgG antibody that is bound to the PF4-heparin complex also binds to platelet Fc $\gamma$ receptors, triggering platelet activation and promoting thrombosis. ${ }^{10}$ In VITT, the vaccine is believed to lead to development of an IgG that targets PF4 (as opposed to the PF4-heparin complex), also binding platelet Fc $\gamma$ receptors and activating platelets. ${ }^{7}$ Although IgG does not require heparin to activate platelets in VITT, heparin may aggravate VITT because circulating PF4-heparin complexes may also bind IgG from VITT as from HIT.

It is important to note that the rapid, latex-enhanced HIT immoassay used in our laboratory does not detect IgG from VITT. However, ELISA assays for HIT generally do detect VITTassociated IgG, and should be ordered if VITT is suspected. With our patient, VITT was eventually confirmed with 2 assays that are currently available for HIT: an ELISA for PF4 that screens for IgG from VITT and the platelet serotonin-release assay that confirms VITT. In HIT, both assays detect PF4-heparin-IgG polyanion complexes. In HIT, the serotonin-release assay infers platelet activation by measuring the release of serotonin when patient serum that contains IgG is incubated with heparin. ${ }^{9}$ In VITT, the serotonin-release assay detects anti-PF4 IgG without the addition of heparin. ${ }^{11}$

Intravenous immunoglobulin is a suggested treatment for patients with VITT who have a worrisome clinical presentation such as bleeding and continued thrombus formation, as with

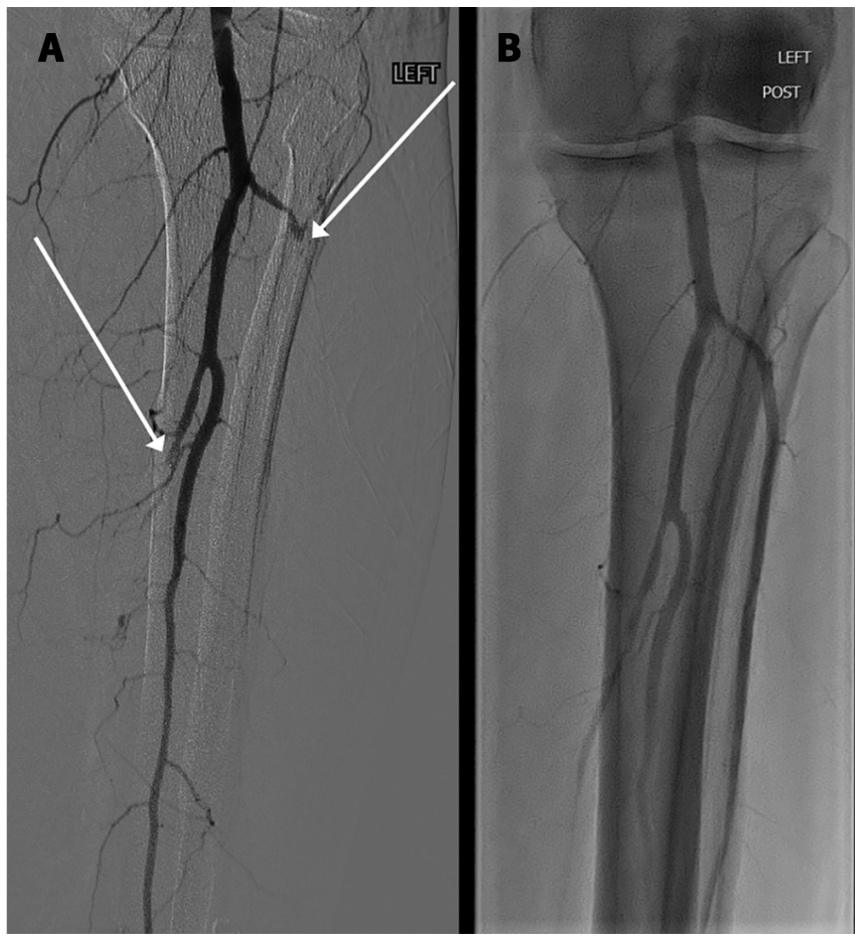

Figure 3: Popliteal and tibial arteriogram of the patient's left leg showing (A) postoperative rethrombosis of the anterior and posterior tibial arteries (arrows), then (B) recanalization after aspiration thrombectomy. 


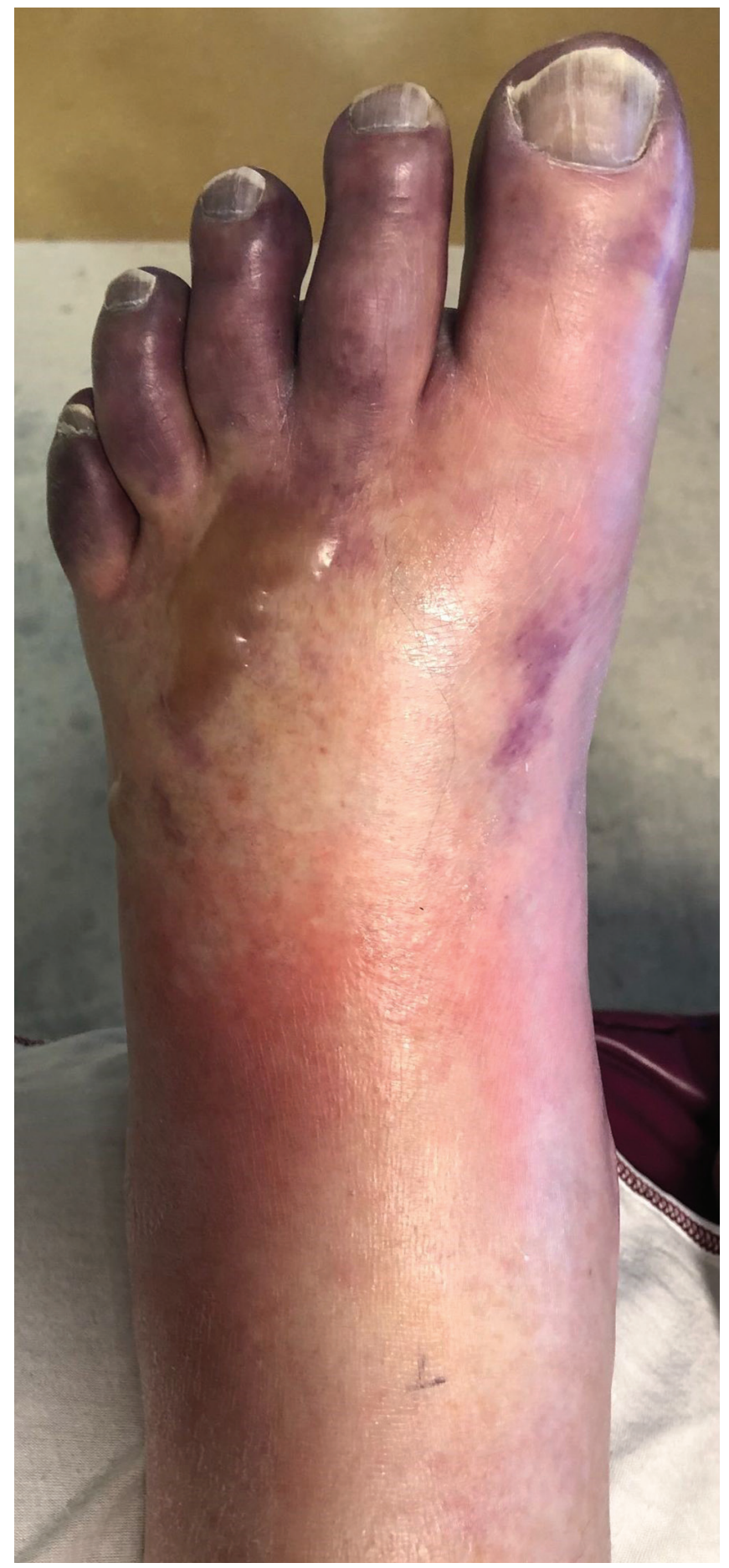

Figure 4: Photograph of the patient's left forefoot showing ischemia on postoperative day 14 .

HIT. We believe this treatment contributed to our patient's eventual improvement. Our decision to use intravenous immunoglobulin was not based on his platelet count, but on the clinical picture of ongoing bleeding and thrombosis that continued despite withdrawal of heparin. In both HIT and VITT, the mechanism of action of intravenous immunoglobulin is believed to be competitive binding of the platelet Fc $\gamma$ receptor by intravenous immunoglobulinderived IgG, which does not activate platelets. ${ }^{12,13}$
Not surprisingly, withdrawal of unfractionated heparin and substitution of fondaparinux 6 days after admission (Figure 2) did not immediately curtail our patient's thrombocytopenia and bleeding. This was likely because of the ability of VITT-associated IgG to bind PF4 without requiring a PF4-heparin complex. ${ }^{7}$ However, clinicians should withdraw heparin (or not use it at all) in patients with VITT, as reduced levels of PF4-heparin complexes can lead to less circulating PF4 antigen.

In our case, we performed emergency surgical thrombectomy because of the severity of limb ischemia. Although we saw excellent early reperfusion of the ischemic extremity, the patient's unrecognized VITT likely led to thrombosis and infarction of the toes, despite a weight-adjusted dose of fondaparinux. Platelet transfusions given to treat bleeding may have worsened thrombosis by providing further reagent for VITT-associated antibodies to trigger clotting. Earlier recognition and treatment of VITT with intravenous immunoglobulin may have lessened thrombosis after what appeared to be effective surgical thrombectomy. Finally, the inability of our latex-enhanced immunoassay for HIT to detect VITT antibodies against PF4 contributed to a delay in diagnosis.

The earliest published cases of VITT described mostly atypical venous thromboses, such as cerebral venous thrombosis and thrombosis of the splanchnic veins, in young healthy women 5-16 days after the first dose of ChAdOx1 nCoV-19 vaccination. ${ }^{2,3}$ In 1 study, all patients with VITT were Norwegian health care workers aged younger than 65 years, $80 \%$ of whom were female; at the time, this population was preferentially vaccinated with ChAdOx1 nCoV-19 in Norway. ${ }^{3}$ These early reports may not accurately portray the true demographic distribution and clinical behaviour of VITT. A recent publication from the United Kingdom is more representative of the general population that has been given the ChAdOx1 $\mathrm{nCoV}$-19 vaccine. It identified VITT in a much broader group of people aged $21-77$ years, $60 \%$ of whom were female. ${ }^{7}$ Our patient was a middle-aged man whose symptoms started 20 days after he received his first dose of vaccine, and he presented with thrombosis in multiple vascular beds. Recent publications from Thrombosis Canada and the International Society on Thrombosis and Hemostasis provide diagnostic criteria and treatment information for VITT. ${ }^{14,15}$ They show that patients with VITT can present with a wide range of thrombotic events and they do not identify any age or gender diagnostic criteria. Two recent reports also describe VITT among patients who received the Ad26.COV2.S vaccine (Johnson \& JohnsonJanssen); ${ }^{6,16}$ the International Society on Thrombosis and Hemostasis identifies both vaccines that use adenovirus vectors (ChAdOx1 nCoV-19 and Ad26.COV2.S) as potential triggers of VITT. ${ }^{15}$

\section{Conclusion}

All physicians should be aware of VITT, given the current use of ChAdOx1 nCoV-19 and Ad26.COV2.S vaccination against SARS-CoV-2. This condition should be considered in any patient presenting with thrombosis and thrombocytopenia after recently receiving a SARS-CoV-2 vaccine that uses an adenovirus vector. The clinical findings of VITT are similar to 
HIT, but rapid immunoassays for HIT, such as the latexenhanced assay, are often falsely negative in patients with VITT. In contrast, ELISA assays for HIT can detect VITTassociated IgG, and the serotonin-release assay confirms VITT if patient serum triggers platelet activation when incubated without heparin. Finally, if VITT is suspected, heparin should not be used and nonheparin anticoagulants, such as fondaparinux, argatroban or direct oral anticoagulants, should be given instead. For patients with intractable bleeding, ongoing thrombosis or other worrisome features, intravenous immunoglobulin can be beneficial.

\section{References}

1. Rutherford RB, Baker JD, Ernst C, et al. Recommended standards for reports dealing with lower extremity ischemia: revised version. J Vasc Surg 1997;26:517-38.

2. Greinacher A, Thiele T, Warkentin TE, et al. Thrombotic thrombocytopenia after ChAdOx1 nCov-19 vaccination. N Engl J Med 2021 Apr. 9 [Epub ahead of print]. doi: 10.1056/NEJMoa2104840.

3. Schultz NH, Sørvoll IH, Michelsen AE, et al. Thrombosis and thrombocytopenia after ChAdOx1 nCoV-19 vaccination. N Engl J Med 2021 Apr. 9 [Epub ahead of print]. doi: 10.1056/NEJMoa2104882.

4. Mehta $P R$, Mangion SA, Benger $M$, et al. Cerebral venous sinus thrombosis and thrombocytopenia after COVID-19 vaccination - a report of two UK cases. Brain Behav Immun 2021 Apr. 20 [Epub ahead of print]. doi: 10.1016/j.bbi .2021.04.006

5. Wolf ME, Luz B, Niehaus L, et al. Thrombocytopenia and intracranial venous sinus thrombosis after "COVID-19 Vaccine AstraZeneca" exposure. J Clin Med 2021;10:1599.

6. Muir K-L, Kallam A, Koepsell SA, et al. Thrombotic thrombocytopenia after Ad26.COV2.S vaccination. N Engl J Med 2021 Apr. 14 [Epub ahead of print]. doi: 10.1056/NEJMc2105869.

7. Scully M, Singh D, Lown R, et al. Pathologic antibodies to platelet factor 4 after ChAdOx1 nCoV-19 vaccination. N Engl J Med 2021 Apr. 16 [Epub ahead of print]. doi: 10.1056/NEJMoa2105385

8. Warkentin TE, Sheppard J-I, Linkins L-A, et al. Performance characteristics of an automated latex immunoturbidimetricassay [HemosIL $\left.{ }^{\circledR} H I T-A b(P F 4-H)\right]$ for the diagnosis of immune heparin-induced thrombocytopenia. Thromb Res 2017;153:108-17.

9. Sheridan D, Carter C, Kelton JG. A diagnostic test for heparin-induced thrombocytopenia. Blood 1986;67:27-30.

10. Hogan M, Berger JS. Heparin-induced thrombocytopenia (HIT): review of incidence, diagnosis, and management. Vasc Med 2020;25:160-73.

11. Nazy I, Sachs UJ, Arnold DM, et al. Recommendations for the clinical and laboratory diagnosis of vaccine-induced immune thrombotic thrombocytopenia (VITT) for SARS-CoV-2 infections: communication from the ISTH SSC Subcommittee on Platelet Immunology. J Thromb Haemost 2021 Apr. 22 [Epub ahead of print]. doi: 10.1111/jth.15341.

12. Warkentin TE. High-dose intravenous immunoglobulin for the treatment and prevention of heparin-induced thrombocytopenia: a review. Expert Rev Hematol 2019;12:685-98

13. Greinacher A, Liebenhoff U, Kiefel V, et al. Heparin-associated thrombocytopenia: the effects of various intravenous IgG preparations on antibody mediated platelet activation - a possible new indication for high dose i.v. IgG. Thromb Haemost 1994;71:641-5.
14. Vaccine-induced prothrombotic immune thrombocytopenia (VIPIT). Whitby: Thrombosis Canada; 2021. Available: https://thrombosiscanada.ca /wp-uploads/uploads/2021/04/51.-Vaccine-induced-prothrobotic-immune -thrombcytopenia_02April2021.pdf (accessed 2021 May 6).

15. The ISTH releases interim guidance on vaccine-induced immune thrombotic thrombocytopenia (VITT) [news release]. Carrboro (NC): International Society on Thrombosis and Haemostasis; 2021 Apr. 20. Available: www.isth.org/ news/561406/ (accessed 2021 May 6).

16. See I, Su JR, Lale A, et al. US case reports of cerebral venous sinus thrombosis with thrombocytopenia after Ad26.COV2.S vaccination, March 2 to April 21 , 2021. JAMA 2021 Apr. 30 [Epub ahead of print]. doi: 10.1001/jama.2021.7517 .

\section{Competing interests: None declared.}

This article has been peer reviewed.

The authors have obtained patient consent.

Affiliations: Section of Vascular Surgery (Jones, Boisvert, Petrasek) and Division of General Internal Medicine (Landry), Peter Lougheed Centre and the Cumming School of Medicine, University of Calgary, Calgary, Alta.

Contributors: All of the authors contributed to the conception and design of the work and data collection. Melissa Jones, Annie Boisvert and Paul Petrasek analyzed the data and drafted the manuscript. All of the authors revised it critically for important intellectual content, gave final approval of the version to be published and agreed to be accountable for all aspects of the work.

Content licence: This is an Open Access article distributed in accordance with the terms of the Creative Commons Attribution (CC BY-NCND 4.0) licence, which permits use, distribution and reproduction in any medium, provided that the original publication is properly cited, the use is noncommercial (i.e., research or educational use), and no modifications or adaptations are made. See: https://creativecommons.org/ licenses/by-nc-nd/4.0/

Acknowledgements: The authors thank Drs. Tania Pannu, Elizabeth Mackay and Jeffrey Shrum for their expertise and assistance with the care of this patient. They also thank the staff at the McMaster University Platelet Immunology Laboratory (Hamilton, Ont.) for testing and test interpretation that confirmed the diagnosis of vaccine-induced immune thrombotic thrombocytopenia.

Correspondence to: Paul Petrasek, paul.petrasek@albertahealthservices.ca

The section Cases presents brief case reports that convey clear, practical lessons. Preference is given to common presentations of important rare conditions, and important unusual presentations of common problems. Articles start with a case presentation (500 words maximum), and a discussion of the underlying condition follows ( 1000 words maximum). Visual elements (e.g., tables of the differential diagnosis, clinical features or diagnostic approach) are encouraged. Consent from patients for publication of their story is a necessity. See information for authors at www.cmaj.ca. 